\title{
CHEMICAL PECULIARITIES, MASS LOSS, AND FINAL EVOLUTION OF AGB STARS IN THE MAGELLANIC CLOUDS
}

\author{
P.R. Wood \\ Mount Stromlo and Siding Spring Observatories \\ Private Bag, Woden P.O., A.C.T. 2606 \\ Australia
}

\section{INTRODUCTION}

The Magellanic Clouds are sufficiently close that evolved stars which exhibit chemical peculiarities and the effects of mass loss can be readily observed. Such objects include carbon stars, $\mathrm{S}$ stars, long-period variables, $\mathrm{OH} / \mathrm{IR}$ stars and planetary nebulae. Because of the relatively well-known distances of the Magellanic Clouds, the intrinsic luminosities of these objects can be accurately determined, in contrast to the situation in the Galaxy where the great majority of asymptotic giant branch (AGB) stars occur in the field population. In this review, observations of AGB stars in the Magellanic Clouds will be discussed with particular reference to those features which can shed light on mass loss and chemical peculiarities resulting from stellar evolution.

\section{CHEMICAL PECULIARITIES IN AGB STARS}

\section{a. Mechanisms for producing abundance peculiarities}

There are four processes which can produce chemical peculiarities in AGB stars: the first, second and third dredge-up events and envelope (or hot-bottom) burning.

The first and second deedge-up events occur prior to the AGB phase when the star moves from core hydrogen or helium burning, respectively, on to the red giant branch. At this time, envelope convection extends downward in mass into regions which have undergone some $\mathrm{CN}$ cycle processing with the result that material enhanced in ${ }^{14} \mathrm{~N}$ and ${ }^{4} \mathrm{He}$ and deficient in ${ }^{12} \mathrm{C}$ is mixed into the envelope. The first and second dredge-up events have been studied in detail by Becker and Iben (1980).

The third dredge-up occurs as a result of the envelope convection extending downward in mass during a helium shell flash, with the result that the products of both the CNO cycle and the 3- $\alpha$ process are brought to the stellar surface (Iben 1975). As a result of the third dredge-up, the stellar surface is enriched in ${ }^{12} \mathrm{C}$ and s-process elements.

The final process which can alter the surface abundances of AGB stars is 
envelope or hot-bottom burning (Scalo, Despain and Ulrich 1975; Iben 1975). In the more massive and luminous AGB stars, envelope convection during the evolutionary phase between helium shell flashes may extend deep enough that the CN cycle is active at the bottom of the convective region. As a result, the entire envelope may be slowly cycled through the $\mathrm{CN}$ cycle resulting in the conversion of ${ }^{12} \mathrm{C}$ to ${ }^{14} \mathrm{~N}$ and ${ }^{1} \mathrm{H}$ to ${ }^{4} \mathrm{He}$.

A detailed review of the above processes is given by Iben and Renzini (1983). Here only effects of the third dredge-up and envelope burning will be considered.

\section{b. Observations of the third dredge-up}

An excellent observational demonstration of the operation of the third dredge-up is exhibited by the intermediate age clusters in the Magellanic Clouds where, going from lower to higher luminosity on the AGB we pass through the sequence of spectral types $M$ to $S$ to $C$ (Bessell, Wood and Lloyd-Evans 1983; Lloyd Evans 1984). Low on the AGB where there have been few or no helium shell flashes, very little 3- $\alpha$ processed material has been dredged up and the AGB stars have essentially normal abundances (apart from the effects of the first and second dredge-up events) and are of spectral type $\mathrm{M}$ (or $\mathrm{K}$ ). However, at the tip of the sequence of oxygen-rich stars in these clusters we find stars whose spectra show strong bands of the s-process element $\mathrm{Zr}$ (these stars are MS or S stars). Model atmosphere calculations by Brett and Bessell (1987) indicate that these strong bands are not due to a temperature effect but are due to real abundance enhancements of $\mathrm{Zr}$. Thus these stars provide direct evidence for the operation of the third dredge-up in oxygen-rich AGB stars.

At luminosities above the MS and S stars in the Magellanic Cloud clusters, we find cool ( $\mathrm{N}$ type) carbon stars in which the third dredge-up has increased the $\mathrm{C} / \mathrm{O}$ ratio to $>1$. There have been many studies of the carbon stars in the Magellanic Clouds; a good review and comparison of this work with theory is given by Iben and Renzini (1983), with some more recent work being found in Lattanzio (1986).

\section{c. Indirect evidence for envelope burning in $A G B$ stars}

It is well known that the carbon stars in the Magellanic Clouds have an upper limit to their luminosity of $\mathrm{M}_{\mathrm{bol}}=-6$ (e.g. Mould and Aaronson 1987). On the other hand, it is also known that oxygen-rich AGB stars in the LMC and SMC extend in luminosity right up to the AGB limit at $M_{\text {bol }}=-7.1$ (Wood, Bessell and Fox 1983; Hughes and Wood 1987). Why are none of these stars carbon stars? It seems that, in the Magellanic Clouds, there is considerable indirect evidence that envelope burning is preventing the formation of carbon stars with $\mathrm{M}_{\text {bol }}<-6$ by converting ${ }^{12} \mathrm{C}$ dredged-up during helium shell flashes into ${ }^{14} \mathrm{~N}$.

The first piece of evidence is the finding of Richer et al (1979) that the brightest carbon stars in the $\mathrm{LMC}$ have strong bands of ${ }^{13} \mathrm{C}$ indicating that a significant amount of $\mathrm{CN}$ cycling has been occurring in the envelopes of these stars. From this result we might conclude that the brightest carbon stars are undergoing envelope burning at a rate which is fast enough to produce a significant amount of ${ }^{13} \mathrm{C}$ but not fast enough to convert much of the dredged-up ${ }^{12} \mathrm{C}$ 
to ${ }^{14} \mathrm{~N}$ and so prevent $\mathrm{C} / \mathrm{O}$ exceeding 1 . In the more luminous $\mathrm{AGB}$ stars, the envelope burning process works fast enough to prevent carbon star formation.

The second piece of evidence for envelope burning in upper AGB stars in the Magellanic Clouds comes from examination of the spectra of SMC long-period variables (LPVs) which are on the AGB and have $\mathrm{M}_{\mathrm{bol}}<-6$. The pulsation (current) masses of these stars are $\sim 3.5 \mathrm{M}_{\odot}$ while the core masses are $\sim 1 \mathrm{M}_{\odot}$, giving an envelope mass of $\sim 2.5 \mathrm{M}_{\odot}$ (Wood, Bessell and Fox 1983). The $\mathrm{O} / \mathrm{H}$ and $\mathrm{C} / \mathrm{O}$ ratios in the gas from which these stars formed were $\sim 10^{-4}$ and $\sim 0.14$, respectively (Dufour 1983). Applying the enrichment/depletion factors for the first and second dredge-ups given by Becker and Iben (1979), we find that C/O $\approx 0.1$ at the beginning of the thermally pulsing AGB phase. Brett and Bessell (1987) have compared the spectra of the above SMC AGB stars with spectra computed from model atmosphere calculations. They found that it was not possible to tell directly from the spectra whether envelope burning had been operating in a thermally pulsing AGB star. The important quantity determining the nature of the stellar spectrum (particularly the strength of TiO bands) is the amount of $16 \mathrm{O}$ not tied up in $\mathrm{CO}$; this quantity can be adjusted to any desired value by altering the relative amounts of dredge-up and envelope burning. Although large increases in ${ }^{14} \mathrm{~N}$ abundance gave rise to stronger $\mathrm{CN}$ bands, the effect was difficult to disentangle from variations in the $\mathrm{C} / \mathrm{O}$ ratio. However, Brett and Bessell (1987) were able to deduce that the AGB stars they looked at have $\mathrm{C} / \mathrm{O} \approx 0.7$. Assuming that there has been no envelope burning on the AGB, this would imply that $\sim 0.0018 \mathrm{M}_{\odot}$ of ${ }^{12} \mathrm{C}$ has been dredged up since the beginning of the thermally pulsing phase. From the calculations of Iben (1977), this amount of dredge-up should occur in only 18 shell flashes, corresponding to only $\sim 2 \times 10^{4}$ years on the AGB during which $M_{\text {bol }}$ would have increased by only $\sim 0.02$ mag. This is such a brief interval in magnitude and time on the AGB (given that the mass loss rates for optically visible LPVs such as these are $<10^{-5} \mathrm{M}_{\odot} \mathrm{yr}^{-1}$ e.g. Knapp and Morris 1985) that it is hard to believe that the stars could not stay there for $50 \%$ longer, by which time there would have been sufficient dredge-up to convert the star to a carbon star. A simple way to allow these stars to remain on the $A G B$ as oxygen-rich stars for a time interval sufficient for their magnitudes to increase by a few tenths or for mass loss to dissipate their envelopes is to have envelope burning occur in them.

In summary, we have two pieces of indirect evidence for the operation of the envelope burning mechanism in upper AGB stars, but no direct measurement of the ${ }^{14} \mathrm{~N}$ abundance in these stars. The importance of the operation of the envelope burning mechanism is that it provides a method of producing primary nitrogen.

\section{MASS LOSS FROM MAGELLANIC CLOUD AGB STARS}

Direct measurements of mass lass rates from AGB stars in the Magellanic Clouds have not yet been made, although the stellar wind has been observed in a number of OH/IR 
stars. However, deductions about the total amount of mass lost from AGB stars in the Magellanic Clouds can be made from observations of long-period variables, the tips of AGB sequences in clusters, AGB luminosity functions, and planetary nebulae. These aspects of mass loss will now be discussed.

\section{a. Long-period variables}

The long-period variables (LPVs) in the Magellanic Clouds provide some of the most definitive evidence available for large-scale mass loss from AGB stars. Termination of the AGB during the LPV phase is clearly demonstrated in Figures 6 and 7 of Wood, Bessell and Fox (1983) (WBF) who show that the optically visible LPVs in the Magellanic Clouds "disappear" from the AGB with a considerable amount of mass still left in their hydrogen-rich envelopes. This disappearance is presumably due to the formation of a thick circumstellar shell which hides the central star from view. WBF find that stars initially less massive than $\sim 5 \mathrm{M}_{\odot}$ eject their envelopes to become planetary nebulae, while the more massive AGB stars reach the AGB limit, corresponding to the core mass attaining the Chandrasekhar limiting mass of 1.4 $\mathbf{M}_{\odot}$, and explode as supernovae (this type of supernova has been designated Type $1 \frac{1}{2}$ by Iben and Renzini 1983). The most massive planetary nebulae produced in this picture is $\sim 2.1 \mathrm{M}_{\odot}$, while the Type $1 \frac{1}{2}$ supernovae have hydrogen envelopes of $\sim 2.1$ to $7 \mathrm{M}_{\odot}$ at explosion.

Given the current prominance of SN1987A, it is interesting note that there are many LPVs in the LMC which are core helium burning supergiants with $M_{b o l}=-8$, close to the luminosity of the progenitor of SN1987A (this is, of course, much more luminous than the AGB limit). The main sequence mass of this progenitor star must have been about $20 \mathrm{M}_{\odot}$ (see contributions in these proceedings), whereas the pulsation masses indicated in Figure 6 of WBF indicate that in the red supergiant stages, LPVs with $M_{\text {bol }}=-8$ have masses in the range $\sim 7$ to $\sim 20 \mathrm{M}_{\odot}$. If these pulsation masses are at all reliable (see WBF) then they indicate that there has been considerable mass loss from massive red supergiants in the LMC. SN1987A should therefore be surrounded by a shell containing $>10 \mathrm{M}_{\odot}$ of mass loss material, although the distance of this shell from the supernova is very uncertain.

Deep searches for LPVs in the LMC (Wood, Bessell and Paltoglou 1985; Reid, Glass and Catchpole 1987) show that the LPV population is dominated by objects of age a few times $10^{9}$ years which, at the time of their exit from the AGB, have envelope mass $\sim 0.35 \mathrm{M}_{\odot}$, core mass $\sim 0.65 \mathrm{M}_{\odot}$ and $\mathrm{M}_{\mathrm{bol}}=-5$. These objects are presumably the immediate precursors of most of the planetary nebulae in the Magellanic Clouds, and their properties will be compared below with those of the planetary nebulae.

\section{b. $A G B$ tips and $A G B$ luminosity functions}

By observing the luminosities of the brightest AGB stars in clusters of different age in the Magellanic Clouds, the final AGB luminosity can be found as a function of age or initial mass; a recent summary of this work is given by Mould and Aaronson (1987). For clusters of age $2-3 \times 10^{9}$ years, the final AGB luminosity is $M_{b o l}=-5$, in agreement with the 
results for LPVs (although the brightest cluster stars generally are not known variables). However, in the youngest of the rich clusters with AGB stars, which have main sequence turnoff masses of $\sim 5 \mathrm{M}_{\odot}$, the most luminous AGB stars have $\mathrm{M}_{\mathrm{bol}}=-6$. Similarly, Reid and Mould (1985) have obtained luminosity functions for AGB stars away from the bar of the LMC which they interpret as indicating that there seems to be a limit of $M_{b o l}=-6$ for AGB stars.

However, there are two sets of observations which indicate that AGB stars do indeed reach the $A G B$ limit $M_{\text {bol }}=-7.1$. Firstly, the results of WBF and Reid, Glass and Catchpole (1987) for the LPVs show that such stars do reach the AGB limit. Secondly, a luminosity function for red stars in the bar of the LMC obtained by Hughes and Wood (1987) shows that $A G B$ stars extend to $M_{b o l}=-7.1$, although there is a steep fall-off with luminosity above $M_{b o l}=-6$. One explanation for the rapid fall-off in the number of AGB stars above $M_{b o l}$ $=-6$ is provided by Wood and Faulkner (1986) who show that envelope ejection will occur at this point in all but the most massive AGB stars due to the luminosity of the star exceeding the Eddington limit at the base of the hydrogen-rich envelope during the surface luminosity peak of a helium shell flash.

\section{c. OHIIR and IR stars in the Magellanic Clouds}

The IRAS point source catalog has provided many candidate $\mathrm{OH} / \mathrm{IR}$ stars in the LMC, and to a lesser extent, in the SMC. Elias, Frogel and Schwering (1986) have found that two of the LMC IRAS sources are red supergiants with $M_{\text {bol }} \sim-9$ and thick circumstellar shells. Wood, Bessell and Whiteoak (1986) showed that one of these objects was an OH/IR star with $1612 \mathrm{MHz}$ maser emission. In a further study of IRAS sources in the LMC, Wood et al (1987a) have found a further 4 AGB stars in the LMC with thick circumstellar shells, with three of these objects being $\mathrm{OH}$ maser sources. An interesting feature of these observations is that the expansion velocities of the stellar winds giving rise to the mass loss can be derived from the $1612 \mathrm{MHz}$ line profiles. In the LMC AGB stars, the expansion velocities are $\sim 12 \mathrm{~km} \mathrm{~s}^{-1}$, only half the value in similar Galactic OH/IR stars. Wood, Bessell and Whiteoak (1986) argue that this is due to the different metal abundances in the LMC and the Galaxy.

\section{d. Planetary Nebulae}

The masses of planetary nebulae give a direct measure of the amount of rapid mass loss at the end of the AGB phase of evolution. Masses for planetary nebulae have been recently derived by Wood et al (1987b), Meatheringham, Dopita and Morgan (1987), and Barlow (1987), using either observed angular diameters or electron densities, together with $\mathrm{H} \beta$ fluxes. The results of these studies show that planetary nebulae of small size and high density have small ionized masses; the central stars of such objects have presumably only recently left the AGB. There is an evolutionary sequence from these small, dense nebulae to larger, less dense nebulae. The maximum mass of ionized material found in the nebulae is close to the value of $\sim 0.35 \mathrm{M}_{\odot}$ predicted as the mass ejected from the dominant population of intermediate age LPVs in the Magellanic Clouds. 
The luminosities of the central stars derived from $\mathrm{H} \beta$ fluxes (Wood et al 1987b) are all less than $M_{b o l}=-5$, the luminosity at which the LPVs of age 2-3x109 years leave the AGB. This is as expected, as there are a number of processes (such as dust absorption in the nebula, being optically thin) which will act to reduce the derived bolometric luminosities of the central stars.

The combined planetary nebula and OH/IR star data allow one to derive a mean mass loss rate for the "superwind" which leads to planetary nebula formation in the stars of age $2-3 \times 10^{9}$ years in the LMC. The total nebula mass is $\sim 0.35 \mathrm{M}_{\odot}$ : the nebula becomes fully ionized at a radius $R \sim 0.1 \mathrm{pc}$ : this is assumed to occur when the volume occupied by superwind material has become fully ionized: with a superwind expansion velocity of $12 \mathrm{~km} \mathrm{~s}^{-1}$, the time from beginning of the superwind to full ionization of the nebula is thus $\sim 8000$ years: hence, the mean mass loss rate during the superwind mass loss phase is $\sim 0.35 / 8000=4 \times 10^{-5} \mathrm{M}_{\odot} \mathrm{yr}^{-1}$. This value is similar to values derived for Galactic stars (e.g. Renzini 1980). Finally, it is worth noting that the time of 8000 years is roughly the time taken for a planetary nebula nucleus of $0.65 \mathrm{M}_{\diamond}$ (the mass of a typical Magellanic Cloud planetary nebula nucleus as deduced from the LPV data) to evolve from $\mathrm{T}_{\text {eff }}=10^{4} \mathrm{~K}$ to $\sim 10^{5} \mathrm{~K}$ (near the blue end of its evolution) where it can ionize the surrounding nebula (Wood and Faulkner 1987). Hence, it appears that the time taken for the planetary nebula nucleus to evolve from the AGB to $T_{\text {eff }}=10^{4} \mathrm{~K}$ is quite small ( $<$ a few thousand years).

\section{REFERENCES}

Barlow, M.J. 1987, M.N.R.A.S., 227, 590.

Becker, S.A. and Iben, I. 1980, Ap.J., 237, 111.

Bessell, M.S., Wood, P.R. and Lloyd Evans, T. 1983, M.N.R.A.S., 202, 59.

Brett, J.M. and Bessell, M.S. 1987, Astr. Ap., submitted.

Dufour, R.J. 1983, in IAU Symposium 108, "Structure and Evolution of the Magellanic Clouds", eds. S. van den Bergh and K.S. de Boer (Reidel), p.353.

Elias, J.H., Frogel, J.A. and Schwering, P.B.W. 1986, Ap.J., 302, 675.

Hughes, S.M.G. and Wood, P.R. 1987, Proc. Astr. Soc. Australia, in press.

Iben, I. 1975, Ap.J., 196, 525.

Iben, I. 1977, Ap.J., 217, 788.

Iben, I. and Renzini, A. 1983, Ann. Rev. Astr. Ap., 21, 271.

Knapp, G.R. and Morris, M. 1985, Ap.J., 292, 640.

Lattanzio, J.C. 1986, Ap.J., 311, 708.

Lloyd Evans, T. 1984, M.N.R.A.S., 208, 447.

Meatheringham, S.J., Dopita, M.A. and Morgan, D. 1987, Ap.J., submitted.

Mould, J.R. and Aaronson, M. 1987, Ap.J., 303, 10.

Reid, N., Glass, I.S. and Catchpole, R.M. 1987, M.N.R.A.S., in press. 
Reid, N. and Mould, J.R. 1985, Ap.J., 299, 236.

Renzini, A. 1980, in "Physical Processes in Red Giants", eds. I. Iben and A. Renzini (Reidel), p. 431.

Richer, H.B., Olander, N. and Westerlund, B.E. 1979, Ap.J., 230, 724.

Scalo, J.M., Despain, K.M. and Ulrich, R.K. 1975, Ap.J., 196, 805.

Wood, P.R., Bessell, M.S. and Fox, M.W. 1983, Ap.J., 272, 99.

Wood, P.R., Bessell, M.S., Hughes, S.M.G., Whiteoak, J.B., Gardner, F.F. and Otrupcek, R.E. 1987a, in preparation.

Wood, P.R., Bessell, M.S. and Paltoglou, G. 1985, Ap.J., 290, 477.

Wood, P.R., Bessell, M.S. and Whiteoak, J.B. 1986, Ap.J. Letters, 306, L81.

Wood, P.R. and Faulkner, D.J. 1986, Ap.J., 307, 659.

Wood, P.R., Meatheringham, S.J., Dopita, M.A. and Morgan, D.H. 1987b, Ap.J., 320, 178. 